\title{
Connectedness and Continuous Sequences in Finite Topological Spaces
}

\author{
Yatsuka Nakamura \\ Shinshu University \\ Nagano, Japan
}

\begin{abstract}
Summary. First, equivalence conditions for connectedness are examined for a finite topological space (originated in [9]). Secondly, definitions of subspace, and components of the subspace of a finite topological space are given. Lastly, concepts of continuous finite sequence and minimum path of finite topological space are proposed.
\end{abstract}

MML identifier: FINTOP06, version: 7.8.01 4.70.946

The articles [16], [5], [18], [13], [1], [19], [14], [3], [4], [2], [6], [12], [10], [15], [7], [11], [8], and [17] provide the terminology and notation for this paper.

\section{Connectedness and Subspaces}

In this paper $F_{1}$ denotes a non empty finite topology space and $A, B, C$ denote subsets of $F_{1}$.

Let us consider $F_{1}$. One can check that $\emptyset_{\left(F_{1}\right)}$ is connected.

We now state two propositions:

(1) For all subsets $A, B$ of $F_{1}$ holds $(A \cup B)^{b}=A^{b} \cup B^{b}$.

(2) $\left(\emptyset_{\left(F_{1}\right)}\right)^{b}=\emptyset$.

Let us consider $F_{1}$. Observe that $\left(\emptyset_{\left(F_{1}\right)}\right)^{b}$ is empty.

Next we state the proposition

(3) Let $A$ be a subset of $F_{1}$. Suppose that for all subsets $B, C$ of $F_{1}$ such that $A=B \cup C$ and $B \neq \emptyset$ and $C \neq \emptyset$ and $B$ misses $C$ holds $B^{b}$ meets $C$ and $B$ meets $C^{b}$. Then $A$ is connected. 
Let $F_{1}$ be a non empty finite topology space. We say that $F_{1}$ is connected if and only if:

(Def. 1) $\Omega_{\left(F_{1}\right)}$ is connected.

We now state four propositions:

(4) Let $A$ be a subset of $F_{1}$. Suppose $A$ is connected. Let $A_{2}, B_{2}$ be subsets of $F_{1}$. Suppose $A=A_{2} \cup B_{2}$ and $A_{2}$ misses $B_{2}$ and $A_{2}$ and $B_{2}$ are separated. Then $A_{2}=\emptyset_{\left(F_{1}\right)}$ or $B_{2}=\emptyset_{\left(F_{1}\right)}$.

(5) Suppose $F_{1}$ is connected. Let $A, B$ be subsets of $F_{1}$. Suppose $\Omega_{\left(F_{1}\right)}=$ $A \cup B$ and $A$ misses $B$ and $A$ and $B$ are separated. Then $A=\emptyset_{\left(F_{1}\right)}$ or $B=\emptyset_{\left(F_{1}\right)}$.

(6) For all subsets $A, B$ of $F_{1}$ such that $F_{1}$ is symmetric and $A^{b}$ misses $B$ holds $A$ misses $B^{b}$.

(7) Let $A$ be a subset of $F_{1}$. Suppose that

(i) $\quad F_{1}$ is symmetric, and

(ii) for all subsets $A_{2}, B_{2}$ of $F_{1}$ such that $A=A_{2} \cup B_{2}$ and $A_{2}$ misses $B_{2}$ and $A_{2}$ and $B_{2}$ are separated holds $A_{2}=\emptyset_{\left(F_{1}\right)}$ or $B_{2}=\emptyset_{\left(F_{1}\right)}$.

Then $A$ is connected.

Let $T$ be a finite topology space. A finite topology space is said to be a subspace of $T$ if it satisfies the conditions (Def. 2).

(Def. 2)(i) The carrier of it $\subseteq$ the carrier of $T$,

(ii) dom (the neighbour-map of it) $=$ the carrier of it, and

(iii) for every element $x$ of it such that $x \in$ the carrier of it holds (the neighbour-map of it) $(x)=($ the neighbour-map of $T)(x) \cap$ the carrier of it.

Let $T$ be a finite topology space. Note that there exists a subspace of $T$ which is strict.

Let $T$ be a non empty finite topology space. Note that there exists a subspace of $T$ which is strict and non empty.

Let $T$ be a non empty finite topology space and let $P$ be a non empty subset of $T$. The functor $T\lceil P$ yields a strict non empty subspace of $T$ and is defined as follows:

(Def. 3) $\Omega_{T \uparrow P}=P$.

We now state the proposition

(8) For every non empty subspace $X$ of $F_{1}$ such that $F_{1}$ is filled holds $X$ is filled.

Let $F_{1}$ be a filled non empty finite topology space. Note that every non empty subspace of $F_{1}$ is filled.

Next we state a number of propositions:

(9) For every non empty subspace $X$ of $F_{1}$ such that $F_{1}$ is symmetric holds $X$ is symmetric.

(10) For every subspace $X^{\prime}$ of $F_{1}$ holds every subset of $X^{\prime}$ is a subset of $F_{1}$. 
(11) For every subset $P$ of $F_{1}$ holds $P$ is closed iff $P^{\mathrm{c}}$ is open.

(12) Let $A$ be a subset of $F_{1}$. Then $A$ is open if and only if the following conditions are satisfied:

(i) for every element $z$ of $F_{1}$ such that $U(z) \subseteq A$ holds $z \in A$, and

(ii) for every element $x$ of $F_{1}$ such that $x \in A$ holds $U(x) \subseteq A$.

(13) Let $X^{\prime}$ be a non empty subspace of $F_{1}, A$ be a subset of $F_{1}$, and $A_{1}$ be a subset of $X^{\prime}$. If $A=A_{1}$, then $A_{1}{ }^{b}=A^{b} \cap \Omega_{X^{\prime}}$.

(14) Let $X^{\prime}$ be a non empty subspace of $F_{1}, P_{1}, Q_{1}$ be subsets of $F_{1}$, and $P, Q$ be subsets of $X^{\prime}$. Suppose $P=P_{1}$ and $Q=Q_{1}$. If $P$ and $Q$ are separated, then $P_{1}$ and $Q_{1}$ are separated.

(15) Let $X^{\prime}$ be a non empty subspace of $F_{1}, P, Q$ be subsets of $F_{1}$, and $P_{1}$, $Q_{1}$ be subsets of $X^{\prime}$. Suppose $P=P_{1}$ and $Q=Q_{1}$ and $P \cup Q \subseteq \Omega_{X^{\prime}}$. If $P$ and $Q$ are separated, then $P_{1}$ and $Q_{1}$ are separated.

(16) For every non empty subset $A$ of $F_{1}$ holds $A$ is connected iff $F_{1} \uparrow A$ is connected.

(17) Let $F_{1}$ be a filled non empty finite topology space and $A$ be a non empty subset of $F_{1}$. Suppose $F_{1}$ is symmetric. Then $A$ is connected if and only if for all subsets $P, Q$ of $F_{1}$ such that $A=P \cup Q$ and $P$ misses $Q$ and $P$ and $Q$ are separated holds $P=\emptyset_{\left(F_{1}\right)}$ or $Q=\emptyset_{\left(F_{1}\right)}$.

(18) For every subset $A$ of $F_{1}$ such that $F_{1}$ is filled and connected and $A \neq \emptyset$ and $A^{\mathrm{c}} \neq \emptyset$ holds $A^{\delta} \neq \emptyset$.

(19) For every subset $A$ of $F_{1}$ such that $F_{1}$ is filled, symmetric, and connected and $A \neq \emptyset$ and $A^{\mathrm{c}} \neq \emptyset$ holds $A^{\delta_{i}} \neq \emptyset$.

(20) For every subset $A$ of $F_{1}$ such that $F_{1}$ is filled, symmetric, and connected and $A \neq \emptyset$ and $A^{\mathrm{c}} \neq \emptyset$ holds $A^{\delta_{o}} \neq \emptyset$.

(21) For every subset $A$ of $F_{1}$ holds $A^{\delta_{i}}$ misses $A^{\delta_{o}}$.

(22) For every filled non empty finite topology space $F_{1}$ and for every subset $A$ of $F_{1}$ holds $A^{\delta_{o}}=A^{b} \backslash A$.

(23) For all subsets $A, B$ of $F_{1}$ such that $A$ and $B$ are separated holds $A^{\delta_{o}}$ misses $B$.

(24) Let $A, B$ be subsets of $F_{1}$. Suppose $F_{1}$ is filled and $A$ misses $B$ and $A^{\delta_{o}}$ misses $B$ and $B^{\delta_{o}}$ misses $A$. Then $A$ and $B$ are separated.

(25) For every point $x$ of $F_{1}$ holds $\{x\}$ is connected.

Let us consider $F_{1}$ and let $x$ be a point of $F_{1}$. Note that $\{x\}$ is connected.

Let $F_{1}$ be a non empty finite topology space and let $A$ be a subset of $F_{1}$. We say that $A$ is a component of $F_{1}$ if and only if:

(Def. 4) $A$ is connected and for every subset $B$ of $F_{1}$ such that $B$ is connected holds if $A \subseteq B$, then $A=B$.

One can prove the following propositions: 
(26) For every subset $A$ of $F_{1}$ such that $A$ is a component of $F_{1}$ holds $A \neq$ $\emptyset_{\left(F_{1}\right)}$.

(27) If $A$ is closed and $B$ is closed and $A$ misses $B$, then $A$ and $B$ are separated.

(28) If $F_{1}$ is filled and $\Omega_{\left(F_{1}\right)}=A \cup B$ and $A$ and $B$ are separated, then $A$ is open and closed.

(29) For all subsets $A, B, A_{1}, B_{1}$ of $F_{1}$ such that $A$ and $B$ are separated and $A_{1} \subseteq A$ and $B_{1} \subseteq B$ holds $A_{1}$ and $B_{1}$ are separated.

(30) If $A$ and $B$ are separated and $A$ and $C$ are separated, then $A$ and $B \cup C$ are separated.

(31) Suppose that

(i) $\quad F_{1}$ is filled and symmetric, and

(ii) for all subsets $A, B$ of $F_{1}$ such that $\Omega_{\left(F_{1}\right)}=A \cup B$ and $A \neq \emptyset_{\left(F_{1}\right)}$ and $B \neq \emptyset_{\left(F_{1}\right)}$ and $A$ is closed and $B$ is closed holds $A$ meets $B$.

Then $F_{1}$ is connected.

(32) Suppose $F_{1}$ is connected. Let $A, B$ be subsets of $F_{1}$. Suppose $\Omega_{\left(F_{1}\right)}=$ $A \cup B$ and $A \neq \emptyset_{\left(F_{1}\right)}$ and $B \neq \emptyset_{\left(F_{1}\right)}$ and $A$ is closed and $B$ is closed. Then $A$ meets $B$.

(33) If $F_{1}$ is filled and $A$ is connected and $A \subseteq B \cup C$ and $B$ and $C$ are separated, then $A \subseteq B$ or $A \subseteq C$.

(34) Let $A, B$ be subsets of $F_{1}$. Suppose $F_{1}$ is symmetric and $A$ is connected and $B$ is connected and $A$ and $B$ are not separated. Then $A \cup B$ is connected.

(35) For all subsets $A, C$ of $F_{1}$ such that $F_{1}$ is symmetric and $C$ is connected and $C \subseteq A$ and $A \subseteq C^{b}$ holds $A$ is connected.

(36) For every subset $C$ of $F_{1}$ such that $F_{1}$ is filled and symmetric and $C$ is connected holds $C^{b}$ is connected.

(37) Suppose $F_{1}$ is filled, symmetric, and connected and $A$ is connected and $\Omega_{\left(F_{1}\right)} \backslash A=B \cup C$ and $B$ and $C$ are separated. Then $A \cup B$ is connected.

(38) Let $X^{\prime}$ be a non empty subspace of $F_{1}, A$ be a subset of $F_{1}$, and $B$ be a subset of $X^{\prime}$. Suppose $F_{1}$ is symmetric and $A=B$. Then $A$ is connected if and only if $B$ is connected.

(39) For every subset $A$ of $F_{1}$ such that $F_{1}$ is filled and symmetric and $A$ is a component of $F_{1}$ holds $A$ is closed.

(40) Let $A, B$ be subsets of $F_{1}$. Suppose $F_{1}$ is symmetric and $A$ is a component of $F_{1}$ and $B$ is a component of $F_{1}$. Then $A=B$ or $A$ and $B$ are separated.

(41) Let $A, B$ be subsets of $F_{1}$. Suppose $F_{1}$ is filled and symmetric and $A$ is a component of $F_{1}$ and $B$ is a component of $F_{1}$. Then $A=B$ or $A$ misses $B$. 
(42) Let $C$ be a subset of $F_{1}$. Suppose $F_{1}$ is filled and symmetric and $C$ is connected. Let $S$ be a subset of $F_{1}$. If $S$ is a component of $F_{1}$, then $C$ misses $S$ or $C \subseteq S$.

Let $F_{1}$ be a non empty finite topology space, let $A$ be a non empty subset of $F_{1}$, and let $B$ be a subset of $F_{1}$. We say that $B$ is a component of $A$ if and only if:

(Def. 5) There exists a subset $B_{1}$ of $F_{1} \mid A$ such that $B_{1}=B$ and $B_{1}$ is a component of $F_{1} \uparrow A$.

We now state the proposition

(43) Let $D$ be a non empty subset of $F_{1}$. Suppose $F_{1}$ is filled and symmetric and $D=\Omega_{\left(F_{1}\right)} \backslash A$. Suppose $F_{1}$ is connected and $A$ is connected and $C$ is a component of $D$. Then $\Omega_{\left(F_{1}\right)} \backslash C$ is connected.

\section{Continuous Finite Sequences and Minimum Path}

Let us consider $F_{1}$ and let $f$ be a finite sequence of elements of $F_{1}$. We say that $f$ is continuous if and only if the conditions (Def. 6) are satisfied.

(Def. 6)(i) $1 \leq \operatorname{len} f$, and

(ii) for every natural number $i$ and for every element $x_{1}$ of $F_{1}$ such that $1 \leq i$ and $i<\operatorname{len} f$ and $x_{1}=f(i)$ holds $f(i+1) \in U\left(x_{1}\right)$.

Let us consider $F_{1}$ and let $x$ be an element of $F_{1}$. Observe that $\langle x\rangle$ is continuous.

One can prove the following two propositions:

(44) Let $f$ be a finite sequence of elements of $F_{1}$ and $x, y$ be elements of $F_{1}$. If $f$ is continuous and $y=f(\operatorname{len} f)$ and $x \in U(y)$, then $f \frown\langle x\rangle$ is continuous.

(45) Let $f, g$ be finite sequences of elements of $F_{1}$. Suppose $f$ is continuous and $g$ is continuous and $g(1) \in U\left(f_{\operatorname{len} f}\right)$. Then $f^{\frown} g$ is continuous.

Let us consider $F_{1}$ and let $A$ be a subset of $F_{1}$. We say that $A$ is arcwise connected if and only if the condition (Def. 7) is satisfied.

(Def. 7) Let $x_{1}, x_{2}$ be elements of $F_{1}$. Suppose $x_{1} \in A$ and $x_{2} \in A$. Then there exists a finite sequence $f$ of elements of $F_{1}$ such that $f$ is continuous and $\operatorname{rng} f \subseteq A$ and $f(1)=x_{1}$ and $f(\operatorname{len} f)=x_{2}$.

Let us consider $F_{1}$. Observe that $\emptyset_{\left(F_{1}\right)}$ is arcwise connected.

Let us consider $F_{1}$ and let $x$ be an element of $F_{1}$. One can verify that $\{x\}$ is arcwise connected.

The following three propositions are true:

(46) For every subset $A$ of $F_{1}$ such that $F_{1}$ is symmetric holds $A$ is connected iff $A$ is arcwise connected.

(47) Let $g$ be a finite sequence of elements of $F_{1}$ and $k$ be a natural number. If $g$ is continuous and $1 \leq k$, then $g \uparrow k$ is continuous. 
(48) Let $g$ be a finite sequence of elements of $F_{1}$ and $k$ be an element of $\mathbb{N}$. If $g$ is continuous and $k<\operatorname{len} g$, then $g_{\downarrow k}$ is continuous.

Let us consider $F_{1}$, let $g$ be a finite sequence of elements of $F_{1}$, let $A$ be a subset of $F_{1}$, and let $x_{1}, x_{2}$ be elements of $F_{1}$. We say that $g$ is minimum path in $A$ between $x_{1}$ and $x_{2}$ if and only if the conditions (Def. 8) are satisfied.

(Def. 8)(i) $g$ is continuous,

(ii) $\operatorname{rng} g \subseteq A$,

(iii) $g(1)=x_{1}$,

(iv) $g($ len $g)=x_{2}$, and

(v) for every finite sequence $h$ of elements of $F_{1}$ such that $h$ is continuous and $\operatorname{rng} h \subseteq A$ and $h(1)=x_{1}$ and $h($ len $h)=x_{2}$ holds len $g \leq$ len $h$.

One can prove the following propositions:

(49) For every subset $A$ of $F_{1}$ and for every element $x$ of $F_{1}$ such that $x \in A$ holds $\langle x\rangle$ is minimum path in $A$ between $x$ and $x$.

(50) Let $A$ be a subset of $F_{1}$. Then $A$ is arcwise connected if and only if for all elements $x_{1}, x_{2}$ of $F_{1}$ such that $x_{1} \in A$ and $x_{2} \in A$ holds there exists a finite sequence of elements of $F_{1}$ which is minimum path in $A$ between $x_{1}$ and $x_{2}$.

(51) Let $A$ be a subset of $F_{1}$ and $x_{1}, x_{2}$ be elements of $F_{1}$. Given a finite sequence $f$ of elements of $F_{1}$ such that $f$ is continuous and $\operatorname{rng} f \subseteq A$ and $f(1)=x_{1}$ and $f(\operatorname{len} f)=x_{2}$. Then there exists a finite sequence of elements of $F_{1}$ which is minimum path in $A$ between $x_{1}$ and $x_{2}$.

(52) Let $g$ be a finite sequence of elements of $F_{1}, A$ be a subset of $F_{1}, x_{1}, x_{2}$ be elements of $F_{1}$, and $k$ be an element of $\mathbb{N}$. Suppose $g$ is minimum path in $A$ between $x_{1}$ and $x_{2}$ and $1 \leq k$ and $k \leq$ len $g$. Then $g \nmid k$ is continuous and $\operatorname{rng}\left(g\lceil k) \subseteq A\right.$ and $\left(g\lceil k)(1)=x_{1}\right.$ and $\left(g\lceil k)\left(\operatorname{len}(g\lceil k))=g_{k}\right.\right.$.

(53) Let $g$ be a finite sequence of elements of $F_{1}, A$ be a subset of $F_{1}, x_{1}$, $x_{2}$ be elements of $F_{1}$, and $k$ be an element of $\mathbb{N}$. Suppose $g$ is minimum path in $A$ between $x_{1}$ and $x_{2}$ and $k<\operatorname{len} g$. Then $g_{\mid k}$ is continuous and $\operatorname{rng}\left(g_{\downarrow k}\right) \subseteq A$ and $g_{\downarrow k}(1)=g_{1+k}$ and $g_{\downarrow k}\left(\operatorname{len}\left(g_{\downarrow k}\right)\right)=x_{2}$.

(54) Let $g$ be a finite sequence of elements of $F_{1}, A$ be a subset of $F_{1}$, and $x_{1}, x_{2}$ be elements of $F_{1}$. Suppose $g$ is minimum path in $A$ between $x_{1}$ and $x_{2}$. Let $k$ be a natural number. If $1 \leq k$ and $k \leq \operatorname{len} g$, then $g \nmid k$ is minimum path in $A$ between $x_{1}$ and $g_{k}$.

(55) Let $g$ be a finite sequence of elements of $F_{1}, A$ be a subset of $F_{1}$, and $x_{1}, x_{2}$ be elements of $F_{1}$. If $g$ is minimum path in $A$ between $x_{1}$ and $x_{2}$, then $g$ is one-to-one.

Let us consider $F_{1}$ and let $f$ be a finite sequence of elements of $F_{1}$. We say that $f$ is inversely continuous if and only if the conditions (Def. 9) are satisfied.

(Def. 9)(i) $1 \leq$ len $f$, and 
(ii) for all natural numbers $i, j$ and for every element $y$ of $F_{1}$ such that $1 \leq i$ and $i \leq \operatorname{len} f$ and $1 \leq j$ and $j \leq$ len $f$ and $y=f(i)$ and $i \neq j$ and $f(j) \in U(y)$ holds $i=j+1$ or $j=i+1$.

We now state three propositions:

(56) Let $g$ be a finite sequence of elements of $F_{1}, A$ be a subset of $F_{1}$, and $x_{1}$, $x_{2}$ be elements of $F_{1}$. Suppose $g$ is minimum path in $A$ between $x_{1}$ and $x_{2}$ and $F_{1}$ is symmetric. Then $g$ is inversely continuous.

(57) Let $g$ be a finite sequence of elements of $F_{1}, A$ be a subset of $F_{1}$, and $x_{1}$, $x_{2}$ be elements of $F_{1}$. Suppose $g$ is minimum path in $A$ between $x_{1}$ and $x_{2}$ and $F_{1}$ is filled and symmetric and $x_{1} \neq x_{2}$. Then

(i) for every natural number $i$ such that $1<i$ and $i<\operatorname{len} g$ holds $\operatorname{rng} g \cap$ $U\left(g_{i}\right)=\left\{g\left(i-{ }^{\prime} 1\right), g(i), g(i+1)\right\}$

(ii) $\quad \operatorname{rng} g \cap U\left(g_{1}\right)=\{g(1), g(2)\}$, and

(iii) $\quad \operatorname{rng} g \cap U\left(g_{\text {len } g}\right)=\left\{g\left(\right.\right.$ len $\left.g-^{\prime} 1\right), g($ len $\left.g)\right\}$.

(58) Let $g$ be a finite sequence of elements of $F_{1}, A$ be a non empty subset of $F_{1}, x_{1}, x_{2}$ be elements of $F_{1}$, and $B_{0}$ be a subset of $F_{1}\lceil A$. Suppose $g$ is minimum path in $A$ between $x_{1}$ and $x_{2}$ and $F_{1}$ is filled and symmetric and $x_{1} \neq x_{2}$ and $B_{0}=\left\{x_{1}\right\}$. Let $i$ be an element of $\mathbb{N}$. If $i<$ len $g$, then $g(i+1) \in \operatorname{Finf}\left(B_{0}, i\right)$ and if $i \geq 1$, then $g(i+1) \notin \operatorname{Finf}\left(B_{0}, i-{ }^{\prime} 1\right)$.

\section{REFERENCES}

[1] Grzegorz Bancerek. The fundamental properties of natural numbers. Formalized Mathematics, 1(1):41-46, 1990.

[2] Grzegorz Bancerek and Krzysztof Hryniewiecki. Segments of natural numbers and finite sequences. Formalized Mathematics, 1(1):107-114, 1990.

[3] Czesław Byliński. Functions and their basic properties. Formalized Mathematics, 1(1):5565, 1990.

[4] Czesław Byliński. Functions from a set to a set. Formalized Mathematics, 1(1):153-164, 1990.

[5] Czesław Byliński. Some basic properties of sets. Formalized Mathematics, 1(1):47-53, 1990.

[6] Hiroshi Imura and Masayoshi Eguchi. Finite topological spaces. Formalized Mathematics, 3(2):189-193, 1992.

[7] Hiroshi Imura, Masami Tanaka, and Yatsuka Nakamura. Continuous mappings between finite and one-dimensional finite topological spaces. Formalized Mathematics, 12(3):381$384,2004$.

[8] Jarosław Kotowicz. Functions and finite sequences of real numbers. Formalized Mathematics, 3(2):275-278, 1992.

[9] Yatsuka Nakamura. Finite topology concept for discrete spaces. In H. Umegaki, editor, Proceedings of the Eleventh Symposium on Applied Functional Analysis, pages 111-116, Noda-City, Chiba, Japan, 1988. Science University of Tokyo.

[10] Takaya Nishiyama and Yasuho Mizuhara. Binary arithmetics. Formalized Mathematics, 4(1):83-86, 1993.

[11] Beata Padlewska and Agata Darmochwał. Topological spaces and continuous functions. Formalized Mathematics, 1(1):223-230, 1990.

[12] Masami Tanaka and Yatsuka Nakamura. Some set series in finite topological spaces. Fundamental concepts for image processing. Formalized Mathematics, 12(2):125-129, 2004.

[13] Andrzej Trybulec. Subsets of complex numbers. To appear in Formalized Mathematics. 
[14] Andrzej Trybulec. Domains and their Cartesian products. Formalized Mathematics, 1(1):115-122, 1990.

[15] Andrzej Trybulec. Enumerated sets. Formalized Mathematics, 1(1):25-34, 1990.

[16] Andrzej Trybulec. Tarski Grothendieck set theory. Formalized Mathematics, 1(1):9-11, 1990.

[17] Wojciech A. Trybulec. Pigeon hole principle. Formalized Mathematics, 1(3):575-579, 1990.

[18] Zinaida Trybulec. Properties of subsets. Formalized Mathematics, 1(1):67-71, 1990.

[19] Edmund Woronowicz. Relations and their basic properties. Formalized Mathematics, 1(1):73-83, 1990.

Received August 18, 2006 